\title{
Biological Timekeeping: Clocks, Rhythms and Behaviour
}

\author{
edited by Vinod Kumar \\ Springer (India) Pvt. Ltd., 2017 \\ ISBN 978-81-322-3686-3 \\ Hardcover \$55, 655 pp.
}

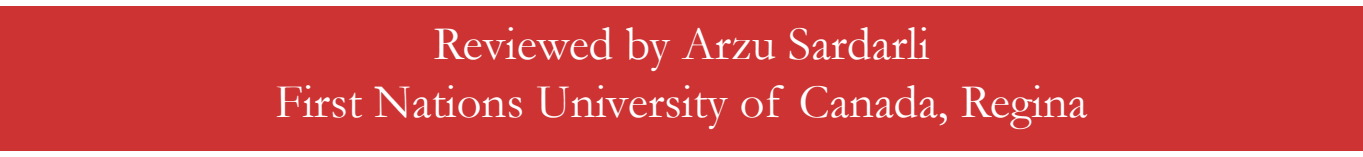

The cyclicity of physical, chemical, biological, and social processes has been the object of intensive study for centuries. The proton-proton cycle was studied as the main mechanism of energy release on stars. At the molecular level, chain chemical processes have a cyclic character. The cell cycle - a sequence of events associated with the multiplication of cells-is essential for the growth, development, and very existence of living organisms. Social processes are not characterized by strict periodicity, although many of them have a cyclic dynamic. Usually, cycling objects interact as parts of a system.

The reviewed book represents the results of studies on the effect of periodicity of physical processes on the features and behaviour of organisms, from bacteria to human. The authors mainly studied the influence of temperature oscillations, the rotation of the Earth around its axis (circadian), and the rotation of the Earth around the Sun (circannual). Some authors analyzed the effect of shorter-period processes (ultradian, tidal).

The book is effectively structured. Each of the thirty-one independent articles constitutes a chapter, and the chapters are grouped into seven parts. Some chapters review the existing literature on circadian, circannual, and other shorter-period rhythms of biological processes; other chapters present new findings.

Part I, "History, Concepts, Evolution, and Basic Features of Biological Clock," contains chapters 1-5 and reviews studies on biological clocks and the main concepts of the developed theories in this field. I would like to make note of chapter 1, written by Drs. William J. Schwartz and Serge Daan, where the authors provide a particularly compelling historical exploration of the ancestry of circadian biology.

In part II, "Animal Clocks: Complexity and Diversity" (chapters 6-12), the authors discuss the cellular and molecular mechanisms regulating circadian rhythms through a large range of organisms: amphibians, insects, fish, reptiles, birds, and mammals. In chapter 11 (Vincent M. Cassone, Jiffin K. Paulose, Clifford E. Harpole, Ye Li, and Melissa Whitfield-Rucker), the authors demonstrate that, unlike mammals, there are three different master clocks in birds that regulate timing in such a way that they produce synchronized events in the daily lives of all birds.

Part III (chapters 13-15), "Human Circadian Rhythms: Entrainment and Sleep Regulation," discusses the mechanisms underlying daily patterns in human physiology and behaviour. The authors describe the importance of circadian entrainment and how the circadian system is organized to 
fulfill this purpose. The mechanisms of delayed sleep phase disorder in humans are also discussed, and its treatment. Assessment of circadian phases are analyzed as an important diagnostic tool.

In part IV (chapters 16-19), "Clock Interactions Within and Between Individual and the Natural World," the authors review studies on the interaction between oscillations with different periods. They analyze the effect of non-photic cues such as temperature, food, anxiety, and induced activities.

In part V (chapters 20-23), "Circadian Clocks, Metabolism, and Immune Functions," circadian deregulation - a mismatch between the external time and the internal circadian time-is analyzed as one of the causes of metabolic disruptions, chronic diseases, and cancer. In chapter 23 (Silke Kiessling and Nicolas Cermakian), the importance of circadian timing for the development of effective cancer therapies is emphasized for humans, rats, and mice.

In part VI (chapters 24-25), "Pineal, Melatonin, and Biological Timekeeping," the authors specify how melatonin (indoleamine hormone) is involved in circadian organization, behavioural and physiological functions, and photoperiodic measurement in birds and other vertebrates. It is also noted that melatonin plays a significant role in daily and seasonal functions, like the immune response.

Part VII (chapters 26-31), "Circannual Rhythms, Photoperiodism, and Seasonal Behaviour," discusses circannual rhythms that anticipate the Earth's annual periodicity. It is shown that the biology of annual cycles in migration, hibernation, and reproduction in birds, mammals, and fishes is associated with the annual rotation of the Earth around the Sun.

The reviewed book makes a significant contribution to the understanding of the role of periodical physical processes on the physiology and behaviour of organisms. The book would potentially benefit from an article or two on the seasonal oscillations of human births. This interesting phenomenon was first recognized approximately 175 years ago (Quetelet 1842), and periodical environmental factors (such as temperature, photoperiod) are considered as possible determinants of the periodicity of human births (He and Earn 2007; Seaver 1985; Trovato and Odynak 1993).

I have no doubt that this book will be incredibly helpful for a wide range of researchers and graduate students: biologists, medical scientists. The chapters relating to human behaviour could be interesting for psychologists and social scientists as well. Some chapters (for instance chapters $1,6,11,13,16,20,24$, and 26) could potentially be relevant for undergraduate students.

\section{References}

He, D., and D.J.D. Earn. 2007. Epidemiological effects of seasonal oscillations in birth rates. Theoretical Population Biology 72:274-91.

Seiver, D.A. 1985. Trend and variation in the seasonality of U.S. fertility, 1947-1976. Demography 22(1):89-100.

Trovato, F., and D. Odynak. 1993. The seasonality of birth in Canada and the provinces, 1881-1989: Theory and analysis. Canadian Studies in Population 20(1):1-41. 